\title{
BMJ Open Rationale and design of a systematic review: effectiveness and acceptance of technology-based psychological interventions in different clinical phases of depression management
}

\author{
Moritz Köhnen, Levente Kriston, Martin Härter, Jörg Dirmaier, Sarah Liebherz
}

To cite: Köhnen M, Kriston L, Härter $\mathrm{M}$, et al. Rationale and design of a systematic review: effectiveness and acceptance of technology-based psychological interventions in different clinical phases of depression management. BMJ Open 2019;9:e028042. doi:10.1136/ bmjopen-2018-028042

- Prepublication history and additional material for this paper are available online. To view these files, please visit the journal online (http://dx.doi org/10.1136/bmjopen-2018028042).

Received 19 November 2018 Revised 20 December 2018 Accepted 22 January 2019

Check for updates

(C) Author(s) (or their employer(s)) 2019. Re-use permitted under CC BY-NC. No commercial re-use. See rights and permissions. Published by BMJ.

Departement of Medical Psychology, University Medical Center Hamburg-Eppendorf, Hamburg, Germany

Correspondence to

Moritz Köhnen;

m.koehnen@uke.de

\section{ABSTRACT}

Introduction Although many effective treatment options exist, depression is still undertreated indicating gaps in the healthcare system. The complementary provision of mental healthcare through technologies (eg, computer, smartphone) has the potential to fill treatment gaps and to overcome access barriers to mental healthcare. Until now, no systematic review integrates the evidence on different technology-based psychological interventions (TBIs) concerning their effectiveness and acceptance in different clinical phases of depression management (bridging waiting periods, acute treatment and aftercare). The aim of this project is to structure evidence on TBIs regarding different phases of depression management, and to determine effectiveness and acceptance for each clinical phase considering both active (eg, face-to-face treatment) and inactive (eg, waitlist) controls as comparators.

Methods and analysis We will include studies on adults with a formal diagnosis of unipolar depression. Treatments delivered by technologies based on scientific psychological theories will be considered as experimental interventions. The primary effectiveness outcome will be depressive symptoms at study endpoint measured by symptom severity rating scales, and the primary acceptance outcome will be dropping out of the study due to any reason. We will consider only randomised controlled trials, which will be identified by key database searches (including Cochrane Central Register of Controlled Trials, Medline, PsycINF0, PSYNDEX, CINAHL) complemented through searches in clinical trial registries (eg, clinicaltrials. gov) and grey literature searches (eg, Open Grey). Two review authors will independently conduct study selection, data extraction and quality assessment of included studies (using the Cochrane Collaboration's tool for assessing risk of bias). Meta-analyses applying random-effect models as well as subgroup, meta-regression and sensitivity analyses will be performed.

Ethics and dissemination Ethics approval is not required for this study, as we conduct research on secondary data. We will disseminate results via peer-reviewed journal publications, presentations on conferences and via plain language summaries.

PROSPERO registration number CRD42016050413; Pre-results.
Strengths and limitations of the study

- The design of this systematic review is methodologically sound (eg, applying a comprehensive literature search for primary and grey literature, and for ongoing trials; including primary studies of all languages; assessing the body of evidence by using the GRADE approach).

- Patient and public involvement will be present in different stages (defining patient relevant outcomes, dissemination of results) of the systematic review.

- Generally, the strict inclusion and exclusion criteria enables transparency, comparability and a focus on high-quality research but disregards studies not meeting these strict criteria (eg, trials applying quasi-randomisation of participants).

- Despite the strict inclusion criteria, the focus of the review is still broad, since it aims to structure available evidence on technology-based intervention in this field. This may result in a large heterogeneity that can only be explained if sufficient evidence is available.

\section{INTRODUCTION}

Depression is a highly prevalent mental disorder, affecting $16 \%-20 \%$ of the general population during their lifetime. ${ }^{1}$ Depression is associated with psychosocial difficulties, ${ }^{2}$ social withdrawal, ${ }^{3}$ increased mortality, ${ }^{4}$ stigmatisation, ${ }^{5}$ as well as high direct societal costs. ${ }^{6}$ According to the $\mathrm{WHO},{ }^{7}$ with more than 300 million affected individuals unipolar depression makes a large contribution to the burden of disease. Considering the high prevalence and the substantial burden of unipolar depression, optimal treatment strategies are essential. Over the past few decades numerous treatments have been developed and proven their effectiveness for unipolar depression, for instance psychological treatments like cognitive behaviour therapy $(\mathrm{CBT}),{ }^{89}$ interpersonal therapy (IPT), ${ }^{8} 10$ pharmacological 
treatments, ${ }^{18}$ as well as combined psychological and pharmacological treatments. ${ }^{18}$ However, depression is only detected in nearly half of all cases when general practitioners diagnose affected individuals. ${ }^{11}$ Additionally, mental disorders are often not treated as only $54 \%$ (major depressive disorder) to $62 \%$ (dysthymia) of people with a lifetime diagnosis of depression report lifetime service use $^{12}$ indicating access barriers to the healthcare system. Access to mental health services for affected individuals is impeded by both structural (eg, lack of availability) and attitudinal (eg, fear of stigma) barriers. ${ }^{13}$

Technology-based psychological interventions (TBIs) are widely seen as an effective complementary source for addressing the issues of accessibility. ${ }^{14}$ TBIs hold the opportunity to reach people who live in remote areas or those with disabilities and without easy access to healthcare services. ${ }^{15-17}$ Furthermore, people who refuse to seek out traditional services, especially those who wish to remain anonymous, may use technology-based mental health services. ${ }^{18}$ These services are also considered as acceptable by healthcare professionals,${ }^{19}$ by lay people ${ }^{19}$ and by users (eg, affected individuals using TBIs). ${ }^{20} 21$ Additionally, it has been shown that computerised CBT (cCBT) is cost effective when compared with usual care, ${ }^{22}$ no treatment ${ }^{23}$ or conventional CBT. ${ }^{23}$ However broad economic evaluations in this research context are still scarce. ${ }^{24}$ In summary, TBIs hold the chance to enhance geographic and time-related flexibility, reduce waiting times, stigmatisation and costs. ${ }^{22}$

TBIs constitute a heterogeneous group of treatments, ranging from unsupported (without a therapist) self-help computer programs to comprehensive psychotherapeutic treatments delivered by a technical medium like telephone or video. Given the great heterogeneity between TBIs we will apply the framework used by Ebert et al to characterise internet and mobile-based psychological interventions by adopting them to a narrower definition of interventions, which, for example, only includes interventions tailored to particular needs of patients with depression. On the basis of this framework, TBIs can be distinguished by application areas (eg, prevention, blended therapy-ie, the combination of face-to-face therapy and technical elements), the technical aspects (eg, via email, short message service [SMS], online chat), the amount of human support (with or without therapeutic support, synchronous or asynchronous feedback), and their theoretical background (eg, evidence-based treatments like CBT). ${ }^{25}$

Many randomised controlled trials (RCTs) were conducted to address the efficacy and effectiveness of TBIs in the treatment of depression. ${ }^{26-28}$ Additionally, there are several systematic reviews examining the efficacy of web-based respectively computerised interventions for depression. ${ }^{24} 29-31$

For instance, a review by Andersson and Cuijpers ${ }^{31}$ found that guided cCBT yielded larger effect sizes $(d=0.61)$ than unguided cCBT $(d=0.25)$ when administered interventions were tested against control groups (eg, waitlist, care as usual). Richards and Richardson ${ }^{29}$ found a moderate $(d=0.56)$ overall effect size of computer-based interventions. At the end of treatment, interventions with therapist support were superior to unsupported interventions when measures of depression outcome were considered. Unsupported interventions yielded considerable dropout rates in comparison with supported interventions. In both reviews, studies applying waitlist controls yielded greater effects than studies using usual care as control groups. ${ }^{29} 31$

However, until now no systematic review integrates the evidence on different TBIs concerning their effectiveness and acceptance in different clinical phases of depression management for bridging waiting periods, for acute treatment and for aftercare. Therefore, current guideline recommendations are limited to the general effectiveness of cCBT. ${ }^{18}$

\section{Objectives}

The aim of our project is:

1. to structure the available evidence of TBIs according to Ebert et $a l^{25}$;

2. to provide data syntheses on the effectiveness of TBIs in different clinical phases of depression management (waiting periods, acute treatment, aftercare);

3. to identify treatment effect modifiers (eg, symptom severity, type of applied intervention);

4. to explore acceptance (eg, dropout rate) and adverse events (eg, symptom aggravation);

5. to provide an evidence base for (guideline) recommendations addressing clinicians, researchers and the general population.

\section{METHODS}

The review will be conducted according to the standards of the Cochrane Collaboration ${ }^{32}$ and will be reported according to the Preferred Reporting Items for Systematic Reviews and Meta-analyses (PRISMA statement). ${ }^{33}$

\section{Inclusion and exclusion criteria}

Types of studies

RCTs, including cross-over and cluster RCTs, will be included in the systematic review. We will exclude uncontrolled and non-randomised controlled studies from this review. No restrictions regarding other design characteristics will be applied.

\section{Types of participants}

\section{Participant characteristics}

Studies with participants of any gender and ethnicity, aged 18 years or above will be considered for inclusion. We will consider trials including participants aged under 18 if the mean age of included participants is $\geq 18$ years.

\section{Diagnosis}

We will consider studies whose participants have a diagnosis of unipolar depression relying on a formal classification system, such as the International Classification of Diseases $(\mathrm{ICD})^{34}$ or the Diagnostic and Statistical Manual 
of Mental Disorders (DSM $)^{35}$-including all versions of these classification systems or standardised diagnostic interviews based on these systems (eg, Composite International Diagnostic Interview Short-Form) ${ }^{36}$ According to the ICD-10, F32.x, F33.x and F34.1 will be considered as unipolar depression. Studies reporting to investigate depressed patients without fulfilling these respective criteria (eg, studies reporting diagnoses on the basis of cut-off values) will be excluded. Studies on participants with a depressive episode in the context of a bipolar disorder will be excluded. Studies including unipolar as well as bipolar depressive participants will be included if data for the unipolar depressive subsample are reported separately. Mixed samples will be included if the proportion of patients with unipolar depression in the sample is $80 \%$ or more. Regarding to the evaluation of TBIs in aftercare, studies whose participants have been diagnosed with depression according to formal diagnostic criteria at the beginning of the acute treatment will be accepted even if the symptoms are currently remitted.

\section{Comorbidities}

Studies involving participants with comorbid mental or somatic conditions will be included if the concurrent condition is not the main focus of the intervention studied (eg, if the concurrent condition serves as an additional inclusion criterion in primary studies, the trial will be excluded).

\section{Setting}

We will consider different clinical phases of depression management as categories supporting to structure available evidence in order to determine effectiveness and acceptance specifically (see also table 1 ):

- waiting period (before acute treatment begins)

- acute treatment

- aftercare

Within different clinical phases of depression management, TBIs can be distinguished concerning their format of implementation in care models. They can be delivered as stand-alone treatments (eg, TBIs substituting face-to-face treatments), as blended treatments (eg, combining TBIs and face-to-face therapy) or as part of stepped care treatments (eg, TBIs as a low intensity intervention targeting either subsyndromal or mild to moderate forms of depression).$^{25}$ Studies conducted in community, primary, secondary or tertiary services will be included in the systematic review regardless of whether they were conducted in in- or outpatient facilities.

\section{Types of interventions}

\section{Experimental intervention}

TBIs, defined as interventions conducted through technical devices based on an explicit psychotherapeutic theory and conducted with or without therapist guidance,

\section{Table 1 Detailed description of relevant comparisons}

\begin{tabular}{|c|c|c|c|}
\hline $\begin{array}{l}\text { Clinical phase of } \\
\text { depression management }\end{array}$ & Healthcare function & Comparisons & $\begin{array}{l}\text { Relevant healthcare context } \\
\text { (examples) }\end{array}$ \\
\hline Waiting periods & $\begin{array}{l}\text { TBI for bridging periods of } \\
\text { waiting for treatment (Bridge- } \\
\text { TBI) }\end{array}$ & - TBI+waitlist vs waitlist & $\begin{array}{l}\text { Depression is diagnosed by the } \\
\text { family doctor and a psychotherapy } \\
\text { possibility or a psychiatrist's } \\
\text { appointment is not available } \\
\text { immediately; TBI is applied to bridge } \\
\text { the waiting period. }\end{array}$ \\
\hline Aftercare & $\begin{array}{l}\text { TBI for maintaining treatment } \\
\text { effects (maintenance-TBI) }\end{array}$ & $\begin{array}{l}\text { TBI vs another TBI } \\
\text { TBI vs psychotherapy } \\
\text { TBI vs pharmacological } \\
\text { treatment } \\
\text { TBI vs combined treatment } \\
\text { TBI vs usual care } \\
\text { TBI vs no treatment }\end{array}$ & $\begin{array}{l}\text { TBI is offered as an aftercare } \\
\text { possibility (eg, as an alternative to } \\
\text { psychotherapeutic, pharmacological } \\
\text { aftercare or no aftercare). }\end{array}$ \\
\hline
\end{tabular}

\footnotetext{
TBI, technology-based psychological intervention.
} 
will be considered. TBIs have to fulfil the following criteria:

- They must be conducted through technical devices like telephone, smartphone, tablet or computer (online or offline). This includes: telephone- or videobased psychotherapy with regular telephone or video calls between health care provider (eg, psychotherapist) and patient, online chat, emails, SMS, apps, as well as computer-, web- or mobile-based programmes.

- They may be carried out independently by the participant (eg, self-help; unguided TBI) or therapist-supported (guided TBI). If therapist-support will be present, the (therapeutic) guidance may be either synchronous (eg, communication in real-time via teleconferencing or Skype) or asynchronous (eg, delayed communication pathways like texting or emails).

- They must be based on a scientific psychological theory (described in detail and/or manualised and/ or referenced).

- They must be (at least partly) tailored to particular needs of patients with depressive symptoms (eg, the interventions aim to reduce negative beliefs, tries to enhance the mood of the participants or includes behavioural activation).

The intervention may comprise self-help programmes, so-called 'prompts' (reinforcement or feedback automatisms), interactive elements (eg, apps to monitor behavioural activation and its influence on mood), serious games or even complex psychotherapeutic programmes (like cCBT). Trials providing only psychoeducational content, patient decision aids, depression management tools (eg, online platforms managing appointments with general practitioners/psychiatrists) as well as tools supporting adherence to drugs will not be considered in our analysis.

\section{Comparator intervention}

Both controlled and comparative effectiveness studies will be included. The comparators may be:

Active control intervention:

- another TBI

- face-to-face psychotherapy

- psychopharmacological treatment

- combined treatment (psychological and pharmacological treatment)

- other active treatments (eg, exercise, relaxation, acupuncture)

Inactive control intervention:

- waitlist

- no-treatment control (only assessments administered to participants)

- attention-placebo/non-specific control (participants receive a treatment that involves non-specific psychosocial factors, for example, social attention, group cohesion)

- usual care
Types of outcome measures

\section{Primary outcomes}

Primary effectiveness outcome

1. Symptoms severity of depression (metric outcome on depression scales: depression at the end of the intervention)

i. the Montgomery-Åsberg Depression Rating Scale $(\text { MADRS })^{37}$;

ii. the Hamilton Depression Rating Scale (HDRS ${ }^{38}$;

iii. the Quick Inventory of Depressive Symptomatology $(\mathrm{QIDS})^{39}$;

iv. the Patient Health Questionnaire-depression scale (PHQ-9) ${ }^{40}$;

v. the Centre of Epidemiologic Studies Depression Scale Revised (CES-D) ${ }^{41}$;

vi. the Beck-Depression-Inventory version I or II (BDI I; BDI II $)^{42} 43$;

vii. the Hospital Anxiety and Depression Scale-depression subscale (HADS-D) ${ }^{44}$;

viii. the Hospital Anxiety and Depression Scale-fullscale (HADS) ${ }^{44}$;

ix. any other reliable and valid depression symptom scale.

Due to the long tradition of depression research, most instruments used in clinical trials are usually psychometrically sound. Such measures will be preferred throughout the review (either referenced and/or sufficient psychometric quality reported).

Primary acceptance/safety outcome

2. Dropout due to any reason.

\section{Secondary outcomes}

3. Remission rate of depression, preferentially defined as

i. no longer fulfilling the formal diagnostic criteria for depression (DSM, ICD);

ii. scoring below the threshold of clinical relevance on a depression symptom rating scale used by the authors (see above: primary outcomes).

4. Response rate of depression, defined as $50 \%$ or greater symptom reduction in any depression rating scale used by the authors.

5. Concerning aftercare studies: Relapse rate of depression (defined as the return of depressive symptoms before full remission has been achieved) or recurrence rate (defined as the (re)appearance of another new episode after full remission has been achieved), preferentially/formally defined/described as:

i. fulfilment of formal diagnostic criteria for depression (DSM, ICD), or as

ii. exceeding a cut-off on a depression symptom rating scale.

6. Metric outcomes on global scales of mental health, for example, Symptom-Checklist-90-Revised (SCL90-R $)^{45}$.

7. (Health related) quality of life, for example, WHO Quality of Life ${ }^{46}$. 
8. Measures of social functioning, for example, Work and Social Adjustment Scale (WSAS) ${ }^{47}$.

9. Measures of treatment satisfaction, for example, Client Satisfaction Questionnaire (CSQ-8) ${ }^{48}$.

10. Dropout due to adverse events.

11. Experiencing any adverse events.

12. Serious adverse events (eg, suicidal behaviour).

\section{Timing of outcome assessment}

The primary outcome time point will be the 'end of intervention' (regardless of the duration of the intervention). Additionally, outcome will be evaluated at the time point ' 1 year after the end of intervention' providing that enough data are available. If 1-year data are not available, we will use data that range between 6 and 18 months after the end of intervention with a preference for the time that was closest to 1 year after the end of intervention.

\section{Hierarchy of outcome measures}

If more than one diagnostic definition and/or depression symptom rating scale will be available, the presented hierarchy according to the UK National Institute for Health and Clinical excellence ${ }^{49}$ will be applied to select measures, starting with: (i) MADRS, (ii) HDRS, (iii) QIDS and so on (see primary effectiveness outcomes).

When considering the secondary outcomes $6-12$, we will not predefine a hierarchy, as we expect that these outcomes will not be assessed by different tools. If authors of included studies used more than one tool (eg, different rating scales evaluating quality of life) to assess the same secondary outcome, we will choose the tool providing the best psychometrically features (regarding reliability, internal consistency, etc).

\section{Search methods for identification of studies \\ Electronic searches}

The following databases will be searched by using relevant subject headings (standard vocabulary; for example, Medical Subject headings), free text terms and search syntax (eg, Boolean logic), tailored to each database (see online supplementary file 1 ):

- Cochrane Central Register of Controlled Trials (CENTRAL)

- OVID Medline

- OVID PsycINFO

- OVID PSYNDEX

- EBSCOhost CINAHL (Complete)

Reviews conducting research in related fields were used to identify appropriate search terms (eg, standard/ controlled vocabulary and keywords). ${ }^{24} 50-52$ PICOS categories (population; intervention; comparator; outcome; study design $)^{32} 53$ served as a framework structuring our systematic search.

We have screened records from CENTRAL roughly and optimised our search strategies prior to running further databases in order to prevent too many irrelevant references. Our search will not be restricted by date, language or publication status.
Complementary searches in clinical trial registers will be conducted to identify ongoing or non-published studies; the databases are as follows:

- Clinicaltrials.gov

- International Clinical Trials Registry Platform

- German Clinical Trial Register (Deutsches Register Klinischer Studien)

\section{Searching other resources}

Grey literature

We will conduct electronic searches in sources of grey literature presented below:

- Open Grey (http://www.opengrey.eu/)

- Trip Database (https://www.tripdatabase.com/)

- ProQuest Dissertations \& Theses Abstract and Indexing (A\&I) (https://search.proquest.com/ pqdt/advanced?accountid=11262)

- ISI Web of Science (specialised registers: Conference Proceedings Citation Index; Conference Proceedings Citation Index-Social Science \& Humanities)

Our search syntax for electronic searches was adopted for electronic searches of grey literature (eg, standardised vocabulary will not be considered in each database).

\section{Reference lists (forward and backward reference search)}

Reference lists of all relevant publications (included studies and relevant systematic reviews) will be searched. Cited reference search on the ISI Web of Science will be performed for all included publications.

\section{Expert contacts/Correspondence}

The first author of all included publications will be contacted for further information regarding published and unpublished trials.

\section{Data collection and analysis}

Selection of studies

Two review authors will independently screen titles and abstracts of 100 records and code them as' retrieve' (eligible or potentially eligible/unclear) or' do not retrieve' (ineligible). We will calculate inter-rater reliability for these (probe) records. If reliability between review authors is sufficiently high $(r \geq 90)$, one review author will screen the remaining records. Records that are labelled as 'unclear' will be reviewed by a second review author. In the case of insufficient reliability between review authors, all records will be seen by two review authors. The selected full text publications will be screened for inclusion by two independent review authors. We will record the specific reason for exclusion according to PICOS criteria for each study. Discrepancies will be resolved by discussion with a third review author. Duplicates will be excluded and multiple reports focusing on the same study will be collated so that each study rather than each report is the unit of interest in the review. The selection process will be displayed by a PRISMA flow diagram. ${ }^{33}$ 


\section{Data extraction and management}

We will develop a data collection sheet, operationalising all characteristics to be extracted. We will pilot the data collection sheet regarding completeness and applicability on at least one study in this review. Study characteristics and outcome data from included studies will be extracted independently by two review authors. We aim to extract the following characteristics:

- general information (eg, year of publication);

- methodological characteristics (eg, information concerning risk of bias assessment according to the Cochrane Collaboration's tool ${ }^{32}$ );

- demographic and clinical sample characteristics (eg, age distribution, psychiatric and/or somatic comorbidities);

- clinical phases of depression management (eg, acute treatment) and formats of implementation in care models (eg, stand-alone treatment);

- treatment characteristics (eg, applied technologies to deliver the intervention, intensity of human support, theoretical foundation of applied treatments [eg, based on CBT, IPT]);

- sample size and study flow (eg, number of randomised participants, number of dropouts per treatment arm);

- primary and secondary outcome data.

Any discrepancies will be resolved by discussion or by consulting a third review author.

\section{Main comparisons}

The main comparisons depend on the clinical phases of depression management and formats of implementation in care models (see table 1). The chosen comparisons were selected on the basis of clinical importance and expected frequency.

\section{Assessment of risk of bias in included studies}

Two review authors will independently assess risk of bias for the included studies-as described in the guidelines of the Cochrane Handbook for Systematic Reviews of Interventions ${ }^{32}$ :

- Random sequence generation

- Allocation concealment

- Blinding of participants and personnel

- Blinding of outcome assessment

- Incomplete outcome data

- Selective outcome reporting

- Other bias

We will assess the risk of bias for each domain as low, high or unclear. In addition, we will justify each risk of bias judgement by providing a quotation from the study combined with a justification for our judgement in the 'Risk of Bias' table. We will resolve any discrepancies by consensus or if necessary by consulting a third review author.

\section{Statistical analysis}

Measures of treatment effect

Continuous data

Mean differences (MD) will be calculated to analyse continuous data, if the included studies use the same outcome measures. If primary studies use different measures to evaluate the outcome, standardised mean differences (SMD) will be computed.

\section{Dichotomous data}

In order to support clinicians and patients to make informed decisions in the clinical context, we will report remission/response rates, overall dropout rates as well as relapse/recurrence rates as relative risks. We will estimate odds ratios for rare outcomes (eg, occurring of adverse events) or endpoints with highly varying baseline rates. In case of time-to event data in the included studies, we will calculate pooled hazard ratios. The number needed to treat will be computed for each of our outcomes to increase interpretability of our findings and support informed decision-making of clinicians. We will enter data presented as a scale with a consistent direction of effect.

\section{Dealing with missing data}

In case of missing or unclear data, we will contact corresponding authors or study sponsors in order to obtain key study characteristics and missing numerical outcome data when possible (eg, when a study is identified as abstract only). We will document all requests and correspondences. If certain outcomes (eg, response rates) are not reported as expected in included studies, we will estimate dichotomous from appropriate metric variables (and vice versa when necessary). ${ }^{32} 54$

Intention-to-treat (ITT) analysis will be used when reported. We will consider whether ITT analysis was computed in the 'Risk of bias' table. When studies will not provide ITT analysis, we will ask corresponding authors for further information. If no further information on ITT data will be available we will impute data if possible (using appropriate methods for calculating imputations ${ }^{55}$ ) or we will use reported data of included studies if imputation is not possible.

\section{Assessment of heterogeneity}

We will investigate statistical heterogeneity in primary studies by using Cochran's Q-test and will quantify it using the $\mathrm{I}^{2}$ statistic. ${ }^{56}$ We will display our results visually as forest plots. The ranges for the interpretation of the $\mathrm{I}^{2}$ statistics will follow the Cochrane Handbook ${ }^{32}$ :

- $0 \%$ to $40 \%$ : might not be important,

- $30 \%$ to $60 \%$ : may represent moderate heterogeneity,

- $50 \%$ to $90 \%$ : may display substantial heterogeneity,

- $75 \%$ to $100 \%$ : considerable heterogeneity.

All $\mathrm{I}^{2}$ values ranging from $50 \%$ to $100 \%$ display a relevant statistical heterogeneity, which should be explored in subsequent analysis. Nevertheless, we will decide on a case-by-case basis if the quantified statistical heterogeneity needs to be explored further as the individual meaning of the detected heterogeneity depends on various factors, not only on the defined thresholds. ${ }^{32} 57$

\section{Assessment of reporting biases}

Possible publication biases and small-study effects will be tested using visual examination of funnel plots for 
the primary outcomes. When considering continuous outcomes measuring intervention effects as mean differences, we will use Egger's test to test for funnel plot asymmetry ${ }^{58}$ Intervention effects displayed as risk ratios, risk differences and standardised mean differences will be examined only visually as there are no (current) well-established tests for asymmetry available. ${ }^{3259}$ We will analyse funnel plot asymmetry for dichotomous outcomes measured as odds ratios by applying the test which was proposed by Harbord et $a l^{60}$.

\section{Data synthesis}

We will run meta-analyses by applying random effects models. ${ }^{61}$ We assume that the primary studies will vary considerably regarding sample, treatment, and methodological characteristics. In addition, we aim to draw conclusions which allow us to generalise beyond the studies included in our meta-analysis. ${ }^{62}$ If clinical and/ or methodological heterogeneity of the included studies proves to be extremely high, a qualitative rather than a quantitative synthesis of the evidence will be performed.

\section{Subgroup analyses}

To identify effect modifiers, we will calculate a priori defined subgroup analyses (in case of categorical variables) or meta-regression analyses (in case of continuous variables), provided that enough studies are available. ${ }^{32}$ We will formally test differences between before-specified subgroups. ${ }^{63-65}$ All meta-regression analyses will be performed using the restricted maximum likelihood method, a recommended random effects approach that accounts for residual between-trial heterogeneity. ${ }^{66} \mathrm{We}$ will run analyses on the following variables grouped into clinical patient characteristics (depression severity, comorbidity) and treatment characteristics (type of applied intervention, intensity of therapist guidance in the intervention group, type of comparator).

\section{Sensitivity analysis}

We will perform sensitivity analyses to explore the robustness of our findings. The sensitivity analyses will focus on primary outcomes. We will carry out the sensitivity analyses on characteristics suspected to bias our findings, as follows:

- Study quality: studies rated with a high or unclear risk of bias (separately for each of the seven assessed domains according to the risk of bias tool of the Cochrane Handbook) will be excluded. Results will be contrasted to those acquired with data from all studies in order to control for possible effects of study quality on pooled effects.

Further relevant sensitivity analyses identified during the review process will be performed, where applicable.

\section{Assessment of the quality of the body of evidence}

We will use the Grading of Recommendations, Assessments, Development and Evaluation (GRADE) approach to assess the quality of the body of evidence ${ }^{67}$ for the primary outcomes. The quality of the body of evidence according to GRADE distinguishes four different categories: high, moderate, low, very low. GRADE allows to assess whether there is confidence in estimated effects of our review $^{68}$ by judging factors like indirectness of evidence, imprecision of results, unexplained heterogeneity and possible publication bias. ${ }^{32} 68$ Results will be displayed in 'Summary of findings tables' following the Cochrane Handbook. $^{32}$

\section{Study status}

Screening for eligibility is anticipated to be completed at the end of January 2019. We expect to finish data extraction at the end of June 2019 and data analyses will start in the middle of July 2019. Additionally, status information is available following the PROSPERO registration (CRD42016050413).

\section{Patient and public involvement}

We will offer workshops targeting people suffering from depression and their relatives to enhance relevance and acceptance of the presented review. A first workshop took place in December 2017, aiming to provide information on systematic reviews and TBIs in general and to discuss, which outcomes were most important from a patient or relative perspective. ${ }^{69}$ By collecting these outcomes (eg, sleep quality, measures of quality of life, daily life functioning), our review may show to which extent patient-relevant outcomes will be reported in included studies. Infrequent consideration of the patient perspective could stimulate future research efforts by expanding research foci on the patient perspective. The second workshop will be conducted at the end of the project in order to discuss the results, the plain language summary, as well as dissemination strategies addressing the general population.

\section{DISSEMINATION}

First, our review will provide a comprehensive summary structuring the empirical evidence on TBIs. ${ }^{25}$ Second, meta-analytical comparisons focusing on clinical phases of depression management will be presented. Third, possible treatment effect modifiers will be identified. Fourth, we will explore acceptance and safety of TBIs.

We will disseminate findings of the review as publications in peer-reviewed journals and in plain language on the e-mental health portal psychenet.de. ${ }^{70}$ Additionally, we will present findings on both national and international conferences, for instance the German Society for Psychiatry, Psychotherapy and Neuropsychiatry (in German: Deutsche Gesellschaft für Psychiatrie und Psychotherapie, Psychosomatik und Nervenheilkunde) and the International Society for Research on Internet Interventions. The first author (MK) will make extracted data available on request after publishing the results, as we aim to foster the traceability of our results and to enable other researches to reanalyse our data. Any changes or deviations from this study protocol will be reported. 
Acknowledgements We thank Norbert Sunderbrink from the Medical Library of the University Medical Center Hamburg-Eppendorf for his support in developing search strategies for primary literature and grey literature. We thank Mareike Dreier for her support in the process of full-text screening and data extraction.

Contributors SL, LK, JD and MH designed the study and applied for funding. SL, MK and LK prepared the detailed study protocol. SL and MK are responsible for data collection and data management. SL, MK and LK will be responsible for data analyses and all authors will contribute to interpretation of the results. MK and SL drafted the manuscript, with important intellectual contributions from LK, MH and JD. All authors read and approved the final manuscript.

Funding The Federal Ministry of Education and Research (in German: Bundesministerium für Bildung und Forschung; BMBF) is funding this study (funding code: $01 \mathrm{KG} 1705$, funding period: $08 / 2017$ to $02 / 2019$ ). The funding institution had no role in the design of this study and will not have any role during its execution, analyses, interpretation of the data, or the decision to submit results.

Competing interests LK and MH have participated in the 2015 revision of the German S3 national clinical practice guideline on the treatment of adults with unipolar depression. MH, SL and JD are licensed psychotherapists. SL is additionally employed at the institute for psychotherapy at the UKE which provides psychotherapist training in behavior therapy. MK declares that he has no competing interests.

\section{Patient consent for publication Not required}

Provenance and peer review Not commissioned; peer reviewed for ethical and funding approval prior to submission.

Open access This is an open access article distributed in accordance with the Creative Commons Attribution Non Commercial (CC BY-NC 4.0) license, which permits others to distribute, remix, adapt, build upon this work non-commercially, and license their derivative works on different terms, provided the original work is properly cited, appropriate credit is given, any changes made indicated, and the use is non-commercial. See: http://creativecommons.org/licenses/by-nc/4.0/.

\section{REFERENCES}

1. DGPPN, BÄK, KBV, et al. Version 5 [S3-Guideline/National Disease Management Guidline Unipolar Depression - Long Version, 2nd edition. 2015. Version 5]. In: DGPPN, BÄK, KBV, et al. eds, S3Leitlinie/Nationale VersorgungsLeitlinie Unipolare Depression Langfassung, 2. Auflage. 2nd edition, 2015.

2. Cabello M, Mellor-Marsá B, Sabariego C, et al. Psychosocial features of depression: a systematic literature review. J Affect Disord 2012;141:22-33.

3. Frei A, Ajdacic-Gross V, Rössler W, et al. Auswirkungen von depressiven Störungen auf objektive Lebensqualitätsbereiche [Effects of Depressive Disorders on Objective Life Quality Criteria]. Psychiatr Prax 2004;31:298-303.

4. Chesney E, Goodwin GM, Fazel S. Risks of all-cause and suicide mortality in mental disorders: a meta-review. World Psychiatry 2014;13:153-60.

5. Sartorius N. The economic and social burden of depression. J Clin Psychiatry 2001;62(Suppl.15):8-11.

6. Statistisches Bundesamt. Gesundheit. Krankheitskosten [Health. Sickness costs]. Fachserie 12. Reihe 7.2. Wiesbaden: Statistisches Bundesamt, 2010.

7. World Health Organization, editor. The Global Burden of Disease: 2004 Update. Geneva: WHO Press, 2008.

8. National Collaborating Centre for Mental Health. Depression: the treatment and management of depression in adults (updated edition): The British Psychological Society and The Royal College of Psychiatrists. 2010 http://www.nice.org.uk/nicemedia/live/12329/ 45896/45896.pdf (Accessed 05 Jul 2018).

9. Cuijpers P, Berking M, Andersson G, et al. A meta-analysis of cognitive-behavioural therapy for adult depression, alone and in comparison with other treatments. Can J Psychiatry 2013;58:376-85.

10. de Mello MF, de Jesus Mari J, Bacaltchuk J, et al. A systematic review of research findings on the efficacy of interpersonal therapy for depressive disorders. Eur Arch Psychiatry Clin Neurosci 2005;255:75-82.

11. Mitchell AJ, Vaze A, Rao S. Clinical diagnosis of depression in primary care: a meta-analysis. Lancet 2009;374:609-19.

12. Mack S, Jacobi F, Gerschler A, et al. Self-reported utilization of mental health services in the adult German population-evidence for unmet needs? Results of the DEGS1-Mental Health Module (DEGS1MH). Int J Methods Psychiatr Res 2014;23:289-303.
13. Andrade LH, Alonso J, Mneimneh Z, et al. Barriers to mental health treatment: results from the WHO World Mental Health surveys. Psychol Med 2014;44:1303-17.

14. Christensen $\mathrm{H}$, Hickie IB. E-mental health: a new era in delivery of mental health services. Med J Aust 2010;192:2-3.

15. Anderson $\mathrm{C}$, Henner T, Burkey J. Tablet computers in support of rural and frontier clinical practice. Int J Med Inform 2013;82:1046-58.

16. Benavides-Vaello S, Strode A, Sheeran BC. Using technology in the delivery of mental health and substance abuse treatment in rural communities: a review. J Behav Health Serv Res 2013;40:111-20.

17. Carrard I, Rouget P, Fernández-Aranda F, et al. Evaluation and deployment of evidence based patient self-management support program for Bulimia Nervosa. Int J Med Inform 2006;75:101-9.

18. Townsend L, Gearing RE, Polyanskaya O. Influence of health beliefs and stigma on choosing internet support groups over formal mental health services. Psychiatr Serv 2012;63:370-6.

19. Gun SY, Titov N, Andrews G. Acceptability of Internet treatment of anxiety and depression. Australas Psychiatry 2011;19:259-64.

20. Andrews G, Cuijpers P, Craske MG, et al. Computer therapy for the anxiety and depressive disorders is effective, acceptable and practical health care: a meta-analysis. PLoS One 2010;5:e13196.

21. Rost T, Stein J, Löbner M, et al. User Acceptance of Computerized Cognitive Behavioral Therapy for Depression: Systematic Review. J Med Internet Res 2017;19:e309.

22. Musiat P, Tarrier N. Collateral outcomes in e-mental health: a systematic review of the evidence for added benefits of computerized cognitive behavior therapy interventions for mental health. Psychol Med 2014;44:3137-50.

23. Hedman E, Ljótsson B, Lindefors N. Cognitive behavior therapy via the Internet: a systematic review of applications, clinical efficacy and cost-effectiveness. Expert Rev Pharmacoecon Outcomes Res 2012;12:745-64.

24. Arnberg FK, Linton SJ, Hultcrantz M, et al. Internet-delivered psychological treatments for mood and anxiety disorders: a systematic review of their efficacy, safety, and cost-effectiveness. PLoS One 2014;9:e98118.

25. Ebert DD, Van Daele T, Nordgreen T, et al. Internet- and MobileBased Psychological Interventions: Applications, Efficacy, and Potential for Improving Mental Health. Eur Psychol 2018;23:167-87.

26. Holländare $F$, Johnsson $S$, Randestad $M$, et al. Randomized trial of Internet-based relapse prevention for partially remitted depression. Acta Psychiatr Scand 2011;124:285-94.

27. Perini S, Titov N, Andrews G. Clinician-assisted Internet-based treatment is effective for depression: randomized controlled trial. Aust N Z J Psychiatry 2009;43:571-8.

28. Wright JH, Wright AS, Albano AM, et al. Computer-assisted cognitive therapy for depression: maintaining efficacy while reducing therapist time. Am J Psychiatry 2005;162:1158-64.

29. Richards D, Richardson T. Computer-based psychological treatments for depression: a systematic review and meta-analysis. Clin Psychol Rev 2012;32:329-42.

30. Kaltenthaler E, Parry G, Beverley C, et al. Computerised cognitivebehavioural therapy for depression: systematic review. $\mathrm{Br} J$ Psychiatry 2008;193:181-4.

31. Andersson G, Cuijpers P. Internet-based and other computerized psychological treatments for adult depression: a meta-analysis. Cogn Behav Ther 2009;38:196-205.

32. Higgins JPT, Green S, eds. Cochrane Handbook for Systematic Reviews of Interventions Version 5.1.0 [updated March 2011] : The Cochrane Collaboration. 2011. Available from www.handbook. cochrane.org

33. Moher D, Liberati A, Tetzlaff J, et al. Preferred reporting items for systematic reviews and meta-analyses: the PRISMA statement. Ann Intern Med 2009;151:264-9.

34. World Health Organization. International Statistical Classification of Diseases, 10th Revision (ICD-10). Geneva: World Health Organization, 1992.

35. American Psychiatric Association. Diagnostic and Statistical Manual of Mental Disorders. 5th Edition (DSM-5). Arlington: American Psychiatric Publishing, 2013.

36. Kessler RC, Andrews G, Mroczek D, et al. The World Health Organization Composite International Diagnostic Interview short-form (CIDI-SF). Int J Methods Psychiatr Res 1998;7:171-85.

37. Montgomery SA, Asberg M. A new depression scale designed to be sensitive to change. Br J Psychiatry 1979;134:382-9.

38. Hamilton M. A rating scale for depression. $J$ Neurol Neurosurg Psychiatry 1960;23:56-62.

39. Rush AJ, Trivedi MH, Ibrahim HM, et al. The 16-Item Quick Inventory of Depressive Symptomatology (QIDS), clinician rating (QIDS-C), and self-report (QIDS-SR): a psychometric evaluation in patients with chronic major depression. Biol Psychiatry 2003;54:573-83. 
40. Kroenke K, Spitzer RL, Williams JB. The PHQ-9: validity of a brief depression severity measure. J Gen Intern Med 2001;16:606-13.

41. Radloff LS. The CES-D scale: A self-report depression scale for research in the general population. Appl Psychol Meas 1977;1:385-401.

42. Beck A, Ward C, Mendelson M, et al. An Inventory for Measuring Depression. Arch Gen Psychiatry 1961;4 :561-71.

43. Beck A, Steer R, Brown G. BDI-II, Beck depression inventory: manual. San Antonio, TX: The Psychological Corporation, 1996.

44. Zigmond AS, Snaith RP. The hospital anxiety and depression scale. Acta Psychiatr Scand 1983;67:361-70.

45. Derogatis LR. SCL-90-R: Administration, Scoring \& procedures manual for the Revised Version. Townson: Clin Psychomet Res 1983.

46. Skevington SM, Lotfy M, O'Connell KA. The World Health Organization's WHOQOL-BREF quality of life assessment: Psychometric properties and results of the international field trial. A report from the WHOQOL group. Qual Life Res 2004;13:299-310.

47. Mundt JC, Marks IM, Shear MK, et al. The Work and Social Adjustment Scale: a simple measure of impairment in functioning. $\mathrm{Br}$ J Psychiatry 2002;180:461-4.

48. Attkisson CC, Zwick R. The client satisfaction questionnaire. Psychometric properties and correlations with service utilization and psychotherapy outcome. Eval Program Plann 1982;5:233-7.

49. National Collaborating Centre for Mental Health. Depression: the treatment and management of depression in adults: Full guideline(draft for consultation) 2017 [(draft for consultation)]. 2017 https://www.nice.org.uk/guidance/gid-cgwave0725/documents/ draft-guideline (Accessed 02 May 2018).

50. Bee PE, Bower P, Lovell K, et al. Psychotherapy mediated by remote communication technologies: a meta-analytic review. BMC Psychiatry 2008;8:60.

51. Thabrew H, Stasiak K, Hetrick SE, et al. Psychological therapies for anxiety and depression in children and adolescents with long-term physical conditions. Cochrane Database Syst Rev 2017; Issue 1.

52. Eccleston C, Fisher E, Craig L, et al. Psychological therapies (Internet-delivered) for the management of chronic pain in adults. Cochrane Database Syst Rev 2014;Issue 2:CD010152.

53. Counsell C. Formulating questions and locating primary studies for inclusion in systematic reviews. Ann Intern Med 1997;127:380-7.

54. Meister R, von Wolff A, Kriston L. Odds ratios of treatment response were well approximated from continuous rating scale scores for meta-analysis. J Clin Epidemiol 2015;68:740-51.

55. Mavridis D, Chaimani A, Efthimiou O, et al. Addressing missing outcome data in meta-analysis. Evid Based Ment Health 2014;17:85-9.
56. Higgins JP, Thompson SG, Deeks JJ, et al. Measuring inconsistency in meta-analyses. BMJ 2003;327:557-60.

57. Kriston L. Dealing with clinical heterogeneity in meta-analysis. Assumptions, methods, interpretation. Int $J$ Methods Psychiatr Res 2013;22:1-15.

58. Sterne JA, Egger M, Smith GD. Investigating and dealing with publication and other biases. In: Egger M, Smith GD, Altman DG, eds. Systematic reviews in health care: meta-analysis in context. London: BMJ Publishing Group, 2003:248-82.

59. Sterne JA, Sutton AJ, loannidis JP, et al. Recommendations for examining and interpreting funnel plot asymmetry in meta-analyses of randomised controlled trials. BMJ 2011;343:d4002.

60. Harbord RM, Egger M, Sterne JA. A modified test for small-study effects in meta-analyses of controlled trials with binary endpoints. Stat Med 2006;25:3443-57.

61. DerSimonian R, Laird N. Meta-analysis in clinical trials. Control Clin Trials 1986;7:177-88.

62. Field AP, Gillett R. How to do a meta-analysis. Br J Math Stat Psychol 2010;63:665-94.

63. Bucher HC, Guyatt GH, Griffith LE, et al. The results of direct and indirect treatment comparisons in meta-analysis of randomized controlled trials. J Clin Epidemiol 1997:50:683-91.

64. Deeks JJ, Altman DG, Bradburn MJ. Statistical methods for examining heterogeneity and combining results from several studies in meta-analysis. In: Egger M, Smith GD, Altman DG, eds. Systematic Reviews in Health Care: Meta-Analysis in Context: BMJ Publishing Group, 2008:285-312.

65. Song F, Altman DG, Glenny AM, et al. Validity of indirect comparison for estimating efficacy of competing interventions: empirical evidence from published meta-analyses. BMJ 2003;326:472

66. Thompson SG, Sharp SJ. Explaining heterogeneity in meta-analysis: a comparison of methods. Stat Med 1999;18:2693-708.

67. Guyatt G, Oxman AD, Akl EA, et al. GRADE guidelines: 1. Introduction-GRADE evidence profiles and summary of findings tables. J Clin Epidemiol 2011;64:383-94.

68. Balshem $\mathrm{H}$, Helfand M, Schünemann HJ, et al. GRADE guidelines: 3. Rating the quality of evidence. J Clin Epidemiol 2011;64:401-6.

69. Brütt $A L$, Meister R, Bernges $T$, et al. Patient involvement in a systematic review: Development and pilot evaluation of a patient workshop. Z Evid Fortbild Qual Gesundhwes 2017:127-128:56-61.

70. Dirmaier J, Liebherz S, Sänger S, et al. Psychenet.de: Development and process evaluation of an e-mental health portal. Inform Health Soc Care 2016;41:267-85. 